\begin{tabular}{lcc}
\hline & Leerwert & Probe \\
\hline 0 Min. & 0,916 & 0,940 \\
LDH/PK & & \\
10 Min. & 0,887 & 0,874 \\
15 Min. & 0,885 & 0,872 \\
GK & & \\
25 Min. & 0,879 & 0,759 \\
30 Min. & 0,877 & 0,757 \\
\hline $\mathrm{E}_{\text {Glycerin }}$ & 0,008 & 0,115 \\
$\Delta \mathrm{E}_{\text {korrig. }}$ & & 0,107 \\
\hline
\end{tabular}

\section{Literatur}

1. Wieland, O., Biochem. Z. 329, 313 (1957). - 2. KReutz, F., Klin. Wschr. 40, 362 (1962). - 3. Eggstein, M. und F. Kreutz, Klin. Wschr. 44, 262 (1966). - 4. Sperri, W. M., Lipid Analysis. in: Methods of biochem. Analysis 2, 83, Interscience Publ., New York-London (1955). - FoLCH, J., J. biol. Chemistry 226, 497 (1957); siehe hierzu auch Zöllner, N. und D. EBERHAGEN, Untersuchung und Bestimmung der Lipoide im Blut, S. 36, Springer Berlin (1965). - 5. Schmidt, F. H. und K. v. DaHL, Klin. Wschr. im Druck. - 6. Schmidt, F. H., K. v. Dafil, W. SchNell und F. WILLIG, in Vorbereitung.

Dr. Felix H. Schmidt 68 Mannheim-Waldhof Sandhofer Str. 112-132

\title{
Eine fluorometrische Methode zur Testosteron-Bestimmung im Harn
}

\author{
Von V. Graef, P. Jobst ${ }^{1}$ ) und HJ. Staudinger \\ Aus dem Physiologisch-Chemischen Institut der Universität Gießen (Direktor: Prof. Dr. Hj. Staudinger)
}

(Eingegangen am 31. Januar 1968)

Es wird eine neue Methode zur Bestimmung von Testosteron im Menschen-Harn beschrieben. Nach enzymatischer Hydrolyse und Solvolyse wird der Harnextrakt an einer Säule aus Aluminiumoxid gereinigt. Testosteron wird von Epitestosteron und anderen $\Delta^{4}-3-K e t o-$ steroiden durch Dünnschichtchromatographie getrennt. Es wird durch die empfindliche Fluoreszenz-Reaktion auf Lithiumhydroxid quantitativ bestimmt. Die Methode ist schnell auszuführen und erfordert kleine Harnmengen ( $4 \mathrm{~m} /$ für Männer und $10 \mathrm{~m} /$ für Frauen).

A new method is reported for the determination of testosterone in human urine. After enzymatic hydrolysis and solvolysis the urine extract is purified on a column of aluminia. Testosterone is separated from epitestosterone and other $\Delta^{4}-3$-ketosteroids by thin-layer chromatography. It is measured by the sensitive fluorescence reaction on lithium hydroxide. The method is rapid and requires small urine samples $(4 \mathrm{~m} /$ for men and $10 \mathrm{~m} /$ for women).

Seitdem Schubert (1) 1960 erstmalig Testosteron aus menschlichem Harn isoliert und bestimmt hat, sind auch Methoden zur Bestimmung von Testosteron im Harn veröffentlicht worden. $\mathrm{Da}$ die Testosteron-Ausscheidung mit der Testosteron-Produktion korreliert, ist es sinnvoll, Testosteron im Harn zu bestimmen (2). Trotz Anwendung der verschiedensten Reinigungsverfahren gelang es nur wenigen Autoren (3-13), Testosteron von seinem 17 $\alpha$-Epimeren, Epitestosteron, sauber $z u$ trennen. Aus den Untersuchungen von De Nicola (7), Schubert (14) und Korenman (15) geht hervor, daß die ausgeschiedenen Mengen von Testosteron und Epitestosteron in etwa derselben Größenordnung liegen. Da abex Epitestosteron, verglichen mit Testosteron, nur eine ganz geringe androgene Aktivität besitzt, ist für eine genaue Bestimmung des Testosterons die Trennung der beiden Epimeren notwendig. Für die Endbestimmung bedienen sich einige Autoren der Gaschromatographie, andere benutzen isotopenmarkiertes Testosteron. Diese Methoden erfordern also besondere Apparate, die nicht überall verfügbar sind. Andere Methoden, bei denen das Testosteron bzw. sein Oxydationsprodukt colorimetrisch oder fluorometrisch bestimmt wird, sind oft nicht sehr empfindlich und erfordern daher den Einsatz größerer Harnmengen. Diẹ notwendigen Reinigungsverfahren sind meist sehr langwierig.

1) Stipendiat der Alexander-von-Humboldt-Stiftung.
Nach der von uns beschriebenen Methode läßt sich Testosteron von Epitestosteron durch Dünnschichtchromatographie trennen. Bei einer längeren Laufzeit des Dünnschichtchromatogramms kann man auch Epitestosteron von anderen störenden Steroiden trennen und für sich bestimmen. Zur quantitativen Bestimmung benutzen wir die Fluoreszenzmessung auf Lithiumhydroxid-Preßlingen (16). $\Delta^{4}$-3-Ketosteroide geben auf der Oberfläche eines Lithiumhydroxid-Preßlings eine spezifische und sehr empfindliche Fluoreszenz (17). Diese Reaktion haben wir bereits zur Bestimmung des Aldosterons im Harn verwendet (18).

\section{Methodik}

\section{Reagenzien}

Methanol p. a. (Fa. Riedel-de Haën) wird über eine Füllkörperkolonne destilliert.

Äthylacetat für Chromatographie (Fa. Riedel-de Haën), Chloroform p. a. (Fa. E. Merck, Darmstadt)

$\beta$-Glucuronidase/Arylsulfatase aus Helix pomatia (Fa. C. F. Boehringer, Mannheim)

Aluminiumoxid: neutral, Akt. II (Fa. Gebr. Giulini, Ludvigshafen)

Aluminiumoxid $\mathrm{GF}_{254}$ für die Dünnschichtchromatographie (Fa. E. Merck, Darmstadt)

Testosteron (Fa. E. Merck, Darmstadt)

Methylenchlorid: 2/ Methylenchlorid werden mit $200 \mathrm{~m} /$ 0,1proz. Kaliumpermanganat-Lösung, mit $200 \mathrm{~m} / \mathrm{N} \mathrm{NaOH}$ und dreimal mit je $400 \mathrm{~m} /$ Wasser ausgeschüttelt. Nach 24stdg. Trocknen über wasserfreiem Calciumchlorid wird das Lösungsmittel destilliert. 
Acetatpuffer pH 5,2:32,4 g wasserfreies Natriumacetat und $7,15 \mathrm{~m} /$ Eiscssig werden mit dest. Wasser auf $250 \mathrm{~m} /$ aufgefüllt.

Benzol p. a. (Fa. E. Merck, Darmstadt)

Lithiumhydroxid: etwa $98 \% \mathrm{LiOH}$ (Fa. E. Merck, Darmstadt) Hjdrolj'se (nach Baulieu und Jayle (19)):

$4 \mathrm{~m} /$ Männerharn oder $10 \mathrm{~m} /$ Frauenharn werden mit dest. Wasser auf $20 \mathrm{~m} /$ verdünnt. Es wird mit Eisessig auf pH 5,2 angesäuert, mit $50 \mu l$ Glucuronidase/Arylsulfatase und $1 \mathrm{ml}$ Acetatpuffer $\mathrm{pH} 5,2$ versetzt und $24 \mathrm{Stdn}$. bei $37^{\circ}$ inkubiert. Zur Hydrolyse ctwa noch vorhandenen Testosteron-Sulfats schließt sich die Solvolyse an. Dazu säuert man den Harn mit 50proz. Schwefelsäure auf $\mathrm{pH} 1 \mathrm{an}$, löst darin $4 \mathrm{~g}$ Natriumchlorid und extrahiert dreimal mit je $50 \mathrm{~m} /$ Äthylacetat. Die vereinigten Extrakte läßt man 24 Stdn. bei $37^{\circ}$ stehen und schüttelt sie nach dem Abkühlen zweimal mit je $20 \mathrm{~m} / \mathrm{N} \mathrm{NaOH}$ und zweimal mit je $20 \mathrm{ml}$ dest. Wasser aus. Nach Trocknen über wasserfreiem Natriumsulfat dampft man die Lösung im Vakuum ein.

Der Verdampfungsrückstand wird mit zwreimal $2 \mathrm{~m} /$ Chloroform auf eine Säule aus neutralem Aluminiumoxid (Akt. II) $(1 \times 5 \mathrm{~cm})$ gebracht und mit $18 \mathrm{~m} /$ Chloroform nachgespült. Das Eluat dampft man im Vakuum ein. Der Rückstand wird quantitativ mit Methanol in ein spitzes Zentrifugenröhrchen überführt, das Lösungsmittel wird im Vakuum verdampft.

\section{Diinnscbicbtchromatograpbie}

Glasplatten $(20 \times 20 \mathrm{~cm})$, die mit einer $0,25 \mathrm{~mm}$ dicken Schicht Aluminiumoxid $\mathrm{GF}_{254}$ versehen sind, werden $3 \mathrm{Stdn}$. bei $120^{\circ}$ aktiviert. Dann schaltet man den Trockenschrank ab und läßßt die Platten darin erkalten. Sie werden in einem Exsikkator ohne Trockenmittel aufbewahrt. Der Verdampfungsrückstand des Harnextraktes aus den spitzen Zentrifugenröhrchen wird mit $2 \mathrm{mal} 100 \mu \mathrm{l}$ Aceton punktförmig $1,2 \mathrm{~cm}$ vom unteren Rand auf eine Dünnschichtplatte aufgetragen. Am linken und am rechten Rand der Platte trägt man; ebenfalls $1,2 \mathrm{~cm}$ vom unteren Rand entfernt, $2 \mu \mathrm{g}$ Testosteron in $25 \mu \mathrm{l}$ Aceton auf. Die Dünnschichtplatte wird im System Benzol/Methylenchlorid/Äthylacetat (3:1:1 V/V) nach dem Durchlaufverfahren (20) 3 Stdn. entwickelt. Die Testosteron-Flecken an den Rändern der Platte sind unter der UV-Lampe sichtbar. Mit ihrer Hilfe wird die das Testosteron enthaltende Zone des Harnextraktes markiert und ausgekratzt. Das Aluminiumoxid überführt man in ein Zentrifugenröhrchen mit Schliff. Außerdem wird ein gleichgroßer Fleck in Höhe des .Testosterons von einer Stelle der Platte ausgekratzt, wo kein Harnextrakt gelaufen war (Plattenleerwert). Man gibt zu allen Röhrchen je eine Glasperle und schüttelt 5 Min. mit je $2 \mathrm{~m} /$ Methanol in der Schüttelmaschine, zentrifugiert $5 \mathrm{Min}$. bei 3000 U./Min. und pipettiert den Uberstand in spitze Zentrifugenröhrchen. Der Rückstand wird nochmal in der gleichen Weise mit $2 \mathrm{~m} /$ Methanol eluiert. Die vereinigten Eluate werden im Vakuum zur Trockne eingedampft.

\section{Quantitative Bestimmung}

Die Herstellung der Lithiumhydroxid-Preßlinge und die Technik des Auftragens sind in einer früheren Arbeit (16) beschrieben. Der Rückstand in den Spitzröhrchen (Urin-Hauptwert und Plattenleerwert) wird in $100 \mu l$ Chloroform gelöst. Durch Einblasen von Stickstoff engt man die Lösung auf etwa $10 \mu \mathrm{l}$ ein und trägt sie auf einen Lithiumhydroxid-Preßling auf. Das Spitzröhrchen wird noch zweimal mit je $100 \mu l$ Chloroform ausgespült. Die Spüllösungen werden nach Einengen auf $10 \mu \mathrm{l}$ auf den gleichen Preßling aufgetragen. Zur Bestimmung eines Reagenzienleerwertes engt man in einem Spitzröhrchen $100 \mu \mathrm{l}$ Chloroform im Stickstoffstrom auf $10 \mu /$ ein und trägt diese auf einen Preßling auf. Diesen Vorgang wiederholt man, wie beim Hauptwert, noch zweimal. Zur Bestimmung eines Standard-Wertes engt man im Spitzröhrchen eine Lösung von $0,2 \mu \mathrm{g}$ Testosteron in $100 \mu \mathrm{l}$ Chloroform auf $10 \mu \mathrm{l}$ ein, trägt diese Lösung auf einen Prẹling auf und spült das Spitzröhrchen noch zweimal mit je $100 \mu l$ Chloroform aus und trägt die Spüllösungen nach Einengen auf $10 \mu l$ auf einen Preßling auf. Die Fluoreszenz der Preßlinge wird vor und nach $20 \mathrm{Min}$. Exhitzen auf $100^{\circ}$ in einem Photometer
„Eppendorf“ mit Fluoreszenzzusatz gemessen. Die Fluoreszenz für den Hauptwert wird in folgender Weise errechnet:

$$
\begin{aligned}
& F_{\mathbf{H}}=F_{\mathbf{H}(\text { nach) }}-F_{\mathbf{H} \text { (vor) }} \quad \mathrm{F}=\text { relative Intensität der } \\
& \text { Fluoreszenz } \\
& F_{P L}=F_{P L} \text { (nach) }-F_{P L} \text { (vor) } \quad \text { (vor) = vor dem Erhitzen } \\
& F_{\text {Harn-TS }}=F_{H}-F_{P L} \quad \text { (nach) }=\text { nach dem Erhitzen } \\
& \mathrm{H}=\text { Urin-Hauptwert } \\
& \mathrm{PL}=\text { Plattenleerwert } \\
& \text { TS }=\text { Testosteron }
\end{aligned}
$$

Die Fluoreszenz für den Testosteron-Standard (S) wird in folgender Weise korrigiert:

$$
\begin{aligned}
& F_{S}=F_{S(\text { nach })}-F_{S \text { (vor) }}
\end{aligned}
$$

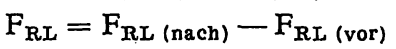

$$
\begin{aligned}
& \mathrm{F}_{\text {Stand.-TS }}=\mathrm{F}_{\mathrm{S}}-\mathrm{F}_{\mathrm{RL}}
\end{aligned}
$$$$
\mathrm{RL}=\text { Reagenzienleerwert }
$$

Bei der Berechnung wird die ermittelte Wiederfindung von $80 \%$ berücksichtigt. In der untersuchten Probe sind

$$
\frac{0,2 \cdot \mathrm{F}_{\text {Harn-TS }}}{\mathrm{F}_{\text {Stand.-TS }}} \cdot \frac{100}{80} \mu \mathrm{g} \text { Testosteron enthalten. }
$$

\section{Ergebnisse}

\section{Spezifität der Metbode}

Abraham und Staudinger (17) konnten zeigen, daß die Fluoreszenzreaktion auf Lithiumhydroxid-Preß-

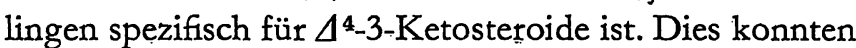
wir bestätigen, z. B. geben $10 \mu \mathrm{g}$ Ätiocholanolon keine Fluoreszenz auf einem Preßling. In Tabelle 1

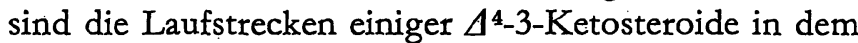

\begin{tabular}{|c|c|}
\hline$\Delta^{4}-3-$ Ketosteroid & Laufstrecke $(\mathrm{cm})$ \\
\hline $\begin{array}{l}\text { Cortisol } \\
\text { Cortison } \\
\text { Corticosteron } \\
11 \text {-Dehydrocorticosteron } \\
6 \beta \text {-Hydroxy-cortisol } \\
\text { Aldosteron } \\
\text { Cortexolon } \\
\text { Desnxycorticosteron } \\
\text { Epitestosteron } \\
\text { 17 } \alpha \text {-Hydroxy-progesteron } \\
\text { Testosteron } \\
\text { Adrenosteron } \\
\text { Androstendion } \\
\text { Progesteron }\end{array}$ & $\begin{array}{l}0 \\
0 \\
0 \\
0 \\
0 \\
0 \\
0 \\
2 \\
7 \\
8 \\
10 \\
13,5 \\
20 \text { (Front) } \\
20 \text { (Front) }\end{array}$ \\
\hline
\end{tabular}
von uns verwendeten.Laufmittelsystem Benzol/Methylenchlorid/Äthylacetat $(3: 1: 1 \mathrm{~V} / \mathrm{V})$ aufgeführt. Daraus ist $\mathrm{zu}$ ersehen, daß Testosteron von anderen $\Delta^{4}$-3 Ketosteroiden sicher getrennt ist.

Tab. 1

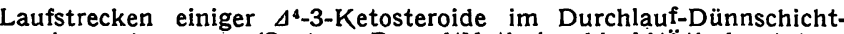
chromatogramm (System Benzol/Methylenchlorid/Äthylacetat chromatogramm (System Benzol/Methy
3:1:1 V/V)

Das aus Harn nach Dünnschichtchromatographie isolierte Testosteron wurde nach VERMEULEN und Verplancke (3) mit Chromtrioxid in Eisessig zu Androstendion oxydiert. Das Oxydationsprodukt sowie authentisches Androstendion wurden auf einer Aluminiumoxid-GF-Platte im System Benzol/Methylenchlorid/Äthylacetat $(3: 1: 1 \mathrm{~V} / \mathrm{V}) 30 \mathrm{Min}$. entwickelt. Das Oxydationsprodukt zeigte bei B̈etrachtung der Platte im UV-Licht nự einen Fleck mit gleichem $R_{\mathrm{F}^{-}}$ Wert wie authentisches Androstendion. - Das aus Harn nach Dünnschichtchromatographie isolièrte 
Testosteron wurde ferner in den Systemen Äthylacetat/Methylenchlorid $(1: 9 \mathrm{~V} / \mathrm{V})$ und Butylacetat/Benzol $(8: 2 \mathrm{~V} / \mathrm{V})$ auf Aluminiumoxid-GF-Platten rechromatographiert. Das aus Harn isolierte Testosteron war bei Betrachtung unter der UV-Lampe einheitlich und hatte den gleichen $R_{\mathrm{F}}$-Wert wie authentisches Testosteron. - Aus dem Harn gewonnenes Testosteron wurde mit konz. Schwefelsäure erhitzt (21), und es wurde das Fluoreszenzspektrum aufgenommen. Es hatte das gleiche Fluoreszenzmaximum bei $538 \mathrm{~nm}$ wie authentisches Testosteron.

\section{Ricbtigkeit der Methode}

Einem zuvor analysierten Harnextrakt wurden 0,3 bzw. 0,6 $\mu \mathrm{g}$ Testosteron zugesetzt, von denen $80 \mathrm{bzw}$. $82 \%$ wiedergefunden wurden. Die wiedergefundenen Mengen sind in Tabelle 2 wiedergegeben.

Tab. 2

Ergebnisse von Wiederfindungsversuchen mit verschiedenen dem Harnextrakt zugesetzten Testosteron-Mengen

\begin{tabular}{ccc}
\hline $\begin{array}{c}\text { zugesetzt } \\
\mu \mathrm{g}\end{array}$ & $\begin{array}{c}\text { Testosteron } \\
\mu \mathrm{g}\end{array}$ & $\begin{array}{c}\text { Wiederfindung } \\
\text { in } \%\end{array}$ \\
\hline 0,30 & 0,24 & 80 \\
0,30 & 0,24 & 80 \\
0,30 & 0,25 & 83 \\
0,30 & 0,22 & $\mathbf{8 3}$ \\
0,30 & 0,25 & 80 \\
& & $\mathbf{8 3}$ \\
\hdashline 0,60 & 0,50 & 65 \\
0,60 & 0,39 & 90 \\
0,60 & 0,54 & $\mathbf{8 3}$ \\
0,60 & 0,50 & $\mathbf{7 3}$ \\
0,60 & 0,44 & $\mathbf{9 7}$ \\
0,60 & 0,58 & $\mathbf{8 2}$ \\
\hline
\end{tabular}

\section{Genauigkeit der Methode}

Ein Mischharn wurde zur Bestimmung der Streuung $7 \mathrm{mal}$ analysiert. Dabei wurde ein Mittelwert von $135 \mu \mathrm{g} / \mathrm{l}$ gefunden. Die Standardabweichung s betrug $\pm 7,4 \mu \mathrm{g}$ ( $\pm 5 \%$ ) (Tab. 3).

Tab. 3

Genauigkeit der Methode Mehrfachbestimmung bei einem Mischharn

\begin{tabular}{c} 
Testosteron im Harn \\
$\mu \mathrm{g} / l$ \\
\hline 136 \\
140 \\
142 \\
142 \\
136 \\
122 \\
129 \\
$\bar{x}=135$ \\
$\mathrm{~s}= \pm \overline{7}, 4( \pm 5 \%)$ \\
\hline
\end{tabular}

\section{Empfindlicbkeit}

Nach der vorliegenden Methode lassen sich noch $0,05 \mu \mathrm{g}$ Testosteron in der Probe sicher bestimmen. Diese Menge gibt eine relative Fluoreszenz von 10 Skalenteilen (Hauptwert abzüglich Leerwert).

\section{Normaliverte}

Die bei 15 männlichen und 8 weiblichen normalen Versuchspersonen gefundenen Werte sind in Tabelle 4 zusammengefaßt. Da die Testosteron-Ausscheidung bei Männern vom Lebensalter abhängig ist, wurden die Männer in Gruppen von je einer Lebensdekade zusammengefaßt.

Tab. 4

Testosteron-Ausscheidung bei gesunden Personen

\begin{tabular}{|c|c|c|}
\hline $\begin{array}{c}\text { Männer } \\
\text { Altersgruppe }\end{array}$ & Alter & $\begin{array}{l}\text { Testosteron } \\
\mu \mathrm{g} / 24 \text { Stdn. }\end{array}$ \\
\hline \multirow[t]{2}{*}{ 21-30 Jahre } & $\begin{array}{l}21 \\
22 \\
23 \\
27 \\
28 \\
29 \\
30\end{array}$ & $\begin{array}{r}118 \\
104 \\
57 \\
100 \\
71 \\
49 \\
77\end{array}$ \\
\hline & & $\bar{x}=82 \mathrm{~s}= \pm 26$ \\
\hline \multirow[t]{2}{*}{$31-40$ Jahre } & $\begin{array}{l}31 \\
31 \\
32 \\
32 \\
36 \\
36\end{array}$ & $\begin{array}{l}85 \\
60 \\
58 \\
60 \\
69 \\
42\end{array}$ \\
\hline & & $\bar{x}=62 \quad s= \pm 14$ \\
\hline \multirow[t]{2}{*}{$41-50$ Jahre } & $\begin{array}{l}42 \\
45\end{array}$ & $\begin{array}{l}59 \\
43\end{array}$ \\
\hline & & $\bar{x}=51$ \\
\hline \multirow[t]{2}{*}{ Frauen } & $\begin{array}{l}21 \\
22 \\
23 \\
25 \\
25 \\
28 \\
35 \\
40\end{array}$ & $\begin{array}{r}9 \\
5 \\
11 \\
11 \\
12 \\
12 \\
4 \\
9\end{array}$ \\
\hline & & $\bar{x}=9 \quad s= \pm 3$ \\
\hline
\end{tabular}

\section{Patbologische Harne}

Tabelle 5 enthält die ausgeschiedenen Mengen Testosteron bei einigen Patienten. Bei einem Knaben von 8 Jahren mit Kryptorchismus fanden wir $8 \mu \mathrm{g}$ Testosteron pro 24 Stdn., ein Wert, der wahrscheinlich für dieses Alter als normal angesehen werden darf (22). Bei zwei Frauen mit Virilismus bzw. : adrenogenitalem Syndrom wurden erwartungsgemäß erhöhte Werte gefunden.

Tab. 5

Bestimmung des Testosteron-Gehaltes pathologischer Harne

\begin{tabular}{lrlcc}
\hline Diagnose & Alter & Geschlecht & $\begin{array}{c}\text { Testo- } \\
\text { steron } \\
\mu \mathrm{g} / 24 \text { Stdn. }\end{array}$ & $\begin{array}{c}\text { 17-Keto- } \\
\text { steroide } \\
\text { mg/ } \\
24 \text { Stdn. }\end{array}$ \\
\hline $\begin{array}{l}\text { Virilismus } \\
\text { Klitorishypertrophie }\end{array}$ & $\mathbf{5 7}$ & weibl. & 44 & 6,5 \\
$\begin{array}{l}\text { Adrenogenitales Syn- } \\
\text { drom }\end{array}$ & $\mathbf{9}$ & weibl. & 15 & 3,0 \\
$\begin{array}{l}\text { Kryptorchismus } \\
\text { Hypogonadismus }\end{array}$ & 10 & weibl. & 30 & 52 \\
$\begin{array}{l}\text { Nebenniérenrinden- } \\
\text { Tumor }\end{array}$ & 17 & $\begin{array}{l}\text { männl. } \\
\text { männl. }\end{array}$ & $\mathbf{2 6}$ & 1,7 \\
\hline
\end{tabular}

\section{Diskussion}

Die von uns beschriebene Methode zeichnet sich dadurch aus, daß sich Testosteron aus dem Harn nach enzymatischer Hydrolyse und Solvolyse sowie nach einer Vorreinigung an einer Aluminiumoxid-Säule durch einmalige eindimensionale Dünnschichtchromatographie von anderen Steroiden mit der $\Delta^{4}-3$-KetoGruppierung, insbesondere von Epitestosteron, trennen läßt. Der Arbeitsaufwand ist dabei geringer als bei 
anderen Methoden. Das aus Harn isolierte Testosteron wurde auf seine Reinheit geprüft. Bei der Rechromatographie in zwei anderen Dünnschichtsystemen erwies es sich als rein. Bei der Oxydation wurde nur Androstendion gefunden. Das Schwefelsäure-Fluoreszenzspektrum stimmte mit demjenigen von reinem Testosteron über-

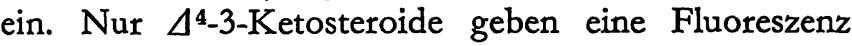
auf der Oberfläche von Lithiumhydroxid-Preßlingen. Keines der bekannteren $\Delta^{4}$-3-Ketosteroide wandert in dem Bereich der Dünnschichtplatte, wo man Testosteron antrifft. Die Methode ist somit für Testosteron spezifisch.

Die Fluoreszenzreaktion ist sehr empfindlich. Noch $0,05 \mu \mathrm{g}$ Testosteron lassen sich in einer Probe bestimmen, so daß diese Reaktion für die. Bestimmung des Testosterons im Plasma aussichtsreich erscheint. Wir fanden im Plasma von zwei Männern 0,60 und $0,47 \mu \mathrm{g}$ Testosteron pro $100 \mathrm{ml}$.
Die von uns ermittelten Normalwerte sind mit den in der Literatur angegebenen vergleichbar. Wir teilten das Kollektiv der Männer in Gruppen von je einer Lebensdekade auf und fanden bei der Gruppe von 21-30 Jahren die höchsten Werte. Mit zunehmendem Alter nimmt die Tesotsteronausscheidung beim Manne langsam ab. Diese Beobachtung deckt sich mit den Untersuchungen von Morer-FARgAs und NowAk̇owskI (2).

Wie aus Tabelle $5 \mathrm{zu}$ ersehen ist, passen die im Harn gefundenen Testosteron-Mengen besser zum klinischen Bild als die Mengen der ausgeschiedenen 17-Ketosteroide. Unsere Methode ist daher für die klinische Diagnostik wertvoll, zumal der Arbeitsaufwand gering ist und die Methode spezifisch und sehr empfindlich ist.

Wir danken Herrn Albert BRÜSTLE für gewissenhafte Mitarbeit. Der eine von uns, P. JoBsT, dankt der Alexander-von-HumboldtStiftung für ein Stipendium.

\section{Literatur}

1. Schubert, K. und K. Wehrberger, Naturwissenschaften 47 , 281 (1960). - 2. Morer-Fargas, F. M. und H. Nowakowski, Acta endocr., K'hvn 49, 443 (1965). - 3. Vermeulen, A. und J. C. M. Verplancke, Steroids 2, 453 (1963). - 4. Korenman, S. G., H. Wilson und M. B. LipseTt, J. Clin. Invest. 42, 1753 (1963). - 5. Sandberg, D. H., N. Ahmad, W. W. Cleveland und K. Savard, Steroids 4, 557 (1964). - 6. Schubert, K. und G. Frankenderg, Hoppe-Seyler's Z. physiol. Chem. 336, 91 (1964). - 7. De Nicola, A., R. I. und E. Forchrelli, PanAmerican Congr. Endocrinol., 6th, Mexico 1965, zit. nach: S. Bernstein, E. W. Cantrall, J. P. Dusza und J. P. Joseph: Steroid Conjugates, Nr. 2120, published by the Chemical Abstracts Service, American Chemical Society, Washington (1966). 8. Rosner, J. M., N. F. Conte, J. H. Briggs, D. Y. Chao, E. M. Sudman und P. H. Forsham, J. Clin. Endocr. Metab., Springfield 25, 95 (1965). - 9. Lim, N. Y. und J. F. Dingman, J. Clin. Endocr. Metab., Springfield 25, 563 (1965). - 10. IsmaIL, A. A. A. und R. A. Harkness, Biochem. J. 99, 717 (1966). - 11. TAMM, J. M. Apostolakis und K. D. Vorgr, Acta endoct., K'hvn 53, 61 (1966). - 12. Wegrenka, L. C., B. F. Bower, J. Shinsako, T. M. Elattar, S. Hane, N. Mimica, E. Demertze, J. E. Stutheit und P. H. Forsham, Analyt. Biochem. 18, 203 (1967). - 13. Brooks, R. V., Steroids 4, 117 (1964). - 14. SchUbert, K., K. Wehrberger und G. Frankenberg, Naturwissenschaften 51, 638 (1964). - 15. Korenman, S. G., H. Wilson und M. B. LIPSETT, J. biol. Chemistry 239, 1004 (1964). - 16. Nowotny, E., R. Abraham und HJ. Staudinger, diese Z. 3, 8 (1965). 17. Abraham, R. und HJ. Staudinger, Z. Naturforsch. 186, 421 (1963). - 18. Nowotny, E. und HJ. Staudinger, diese Z. 4, 203 (1966). - 19. Bauliru, E. E. und M. F. JAYLe, Analyse des Steroides Hormonaux, Tome I, S. 73, Masson et Cie., Paris (1961). 20. Graef, V. und HJ. Staudinger, diese Z. 5, 314 (1967). 21. Abraham, R. und HJ. Staudingeg, diese Z. 2, 16 (1964). 22. KNORR, D., Acta endocr., K'hrn 54, 215 (1967).

Prof. Dr. Hj. Staudinger 63 Gießen

Friedrichstr. 24 